\title{
CORRIGENDUM
}

\section{RIPK1 promotes death receptor-independent caspase-8-mediated apoptosis under unresolved ER stress conditions}

\author{
Y Estornes, MA Aguileta, C Dubuisson, J De Keyser, V Goossens, K Kersse, A Samali, P Vandenabeele and MJM Bertrand
}

Cell Death and Disease (2015) 6, e1798; doi:10.1038/cddis.2015.175; published online 25 June 2015

Correction to: Cell Death and Disease (2014) 5, e1555; doi:10.1038/cddis.2014.523; published online 4 December 2014

Since the publication of this paper the authors have noted an error in Figure 6 . The labelling of the plus and minus symbols in Figure $6 c$ is in the wrong order and does not correlate with the results of the western blot underneath. This has now been rectified, the correct figure is shown below and the corrected article appears online together with this corrigendum.

The authors would like to apologize for any inconvenience this may have caused. 
a

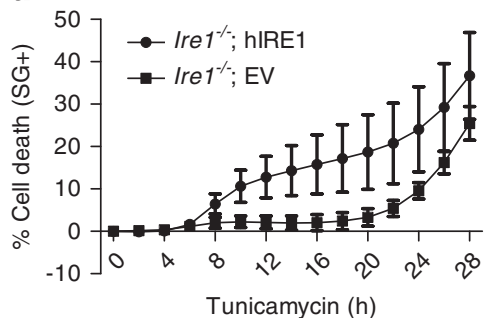

d

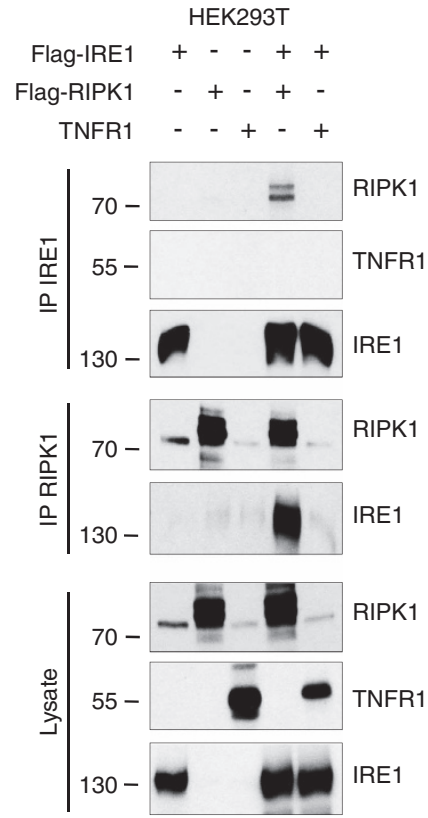

\section{b}

b

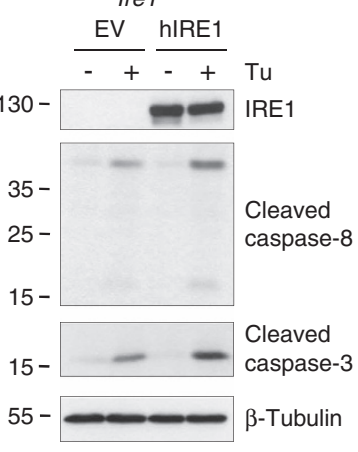

C

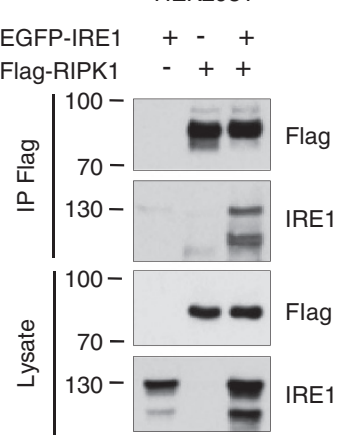

e

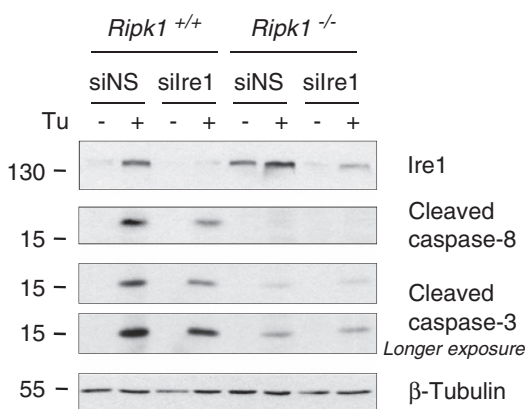

Figure 6 RIPK1 interacts with the pro-apoptotic receptor IRE1. (a and b) Ire $1^{-1-}$ cells reconstituted with an empty vector (EV) or with a vector coding for hIRE1(hIRE1) were stimulated with $1 \mu \mathrm{g} / \mathrm{ml}$ tunicamycin (Tu), and the percentage of cell death was measured in function of time using the Fluostar Omega fluorescence plate reader (a), or cell lysates obtained after $17 \mathrm{~h}$ of stimulation were immunoblotted as indicated (b). (c) HEK293T cells were transiently transfected with plasmids coding for EGFP-hIRE1 and/or Flag-hRIPK1, and RIPK1 was immunprecipitated (IP) using anti-Flag-coated beads. Cell lysates and immunoprecipitates were analyzed by immunoblot as indicated. (d) HEK293T cells were transiently transfected with plasmids coding for Flag-hIRE1, Flag-hRIPK1 and/or hTNFR1, and IRE1 (upper panels) or RIPK1 (middle panels) were immunoprecipitated with antiIRE1 or anti-RIPK1 antibodies, respectively. Cell lysates and immunoprecipitates were analyzed by immunoblot as indicated. (e) Ripk $1^{+/+}$and Ripk $1^{-1-}$ MEFs were transfected with a control non-silencing siRNA (siNS) or targeting Ire1 (silre1) and then exposed to $1 \mu \mathrm{g} / \mathrm{ml}$ Tu for $12 \mathrm{~h}$. The cells were then lysed and immunoblotted as indicated 